\title{
SNR improvement based on piecewise linear interpolation
}

\author{
Pengfei $\mathrm{Xu}^{1}$, Yinjie Jia ${ }^{1,2, *}$
}

\begin{abstract}
Interpolation improves the resolution of the curve. Based on the stationary characteristics of the signal and the nonstationary characteristics of the noise, the theoretical proof indicates that the piecewise linear interpolation can improve the signal- to-noise ratio, which is further confirmed by simulation results.
\end{abstract}

K e y w or d s: signal-to-noise ratio improvement, piecewise linear interpolation, signal enhancement

\section{Introduction}

The function of interpolation is to improve the resolution of the curve to make it is closer to the real waveform. As an important method of function approximation and a practical numerical method, interpolation is widely used in signal processing and graph analysis $[1,2]$. Interpolation methods can be categorized into one- dimensional, twodimensional, and multi-dimensional interpolation. Since this letter mainly deals with one- dimensional signal, this section briefly introduces the one- dimensional interpolation method, which mainly include Lagrange interpolation [3], piecewise linear interpolation, and cubic spline interpolation [4].
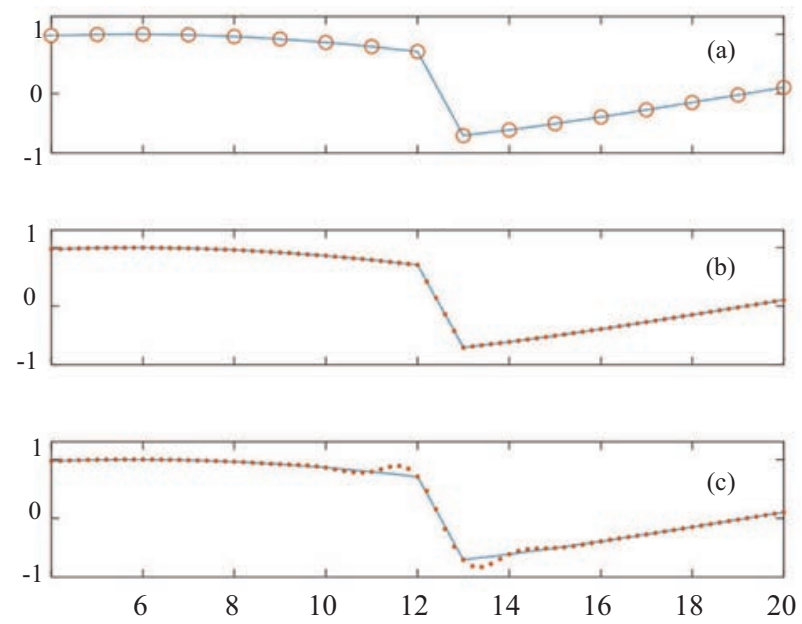

Fig. 1. (a) - original, (b) - piecewise linear interpolation, and (c) - cubic spline interpolation

Lagrange interpolation is a high-order polynomial interpolation. The interpolation function is an analytical expression in the whole interval, and the result curve is smooth. Due to the oscillation phenomenon, the convergence of Lagrange interpolation cannot be guaranteed, so it is generally used in theoretical analysis. Piecewise linear interpolation and cubic spline interpolation belong to low-degree polynomial interpolation with guaranteed convergence. These two interpolation methods are simple, practical and widely used. Specifically, cubic spline interpolation is improved in terms of curve smoothing, but it suffers from difficult error estimation. Piecewise linear interpolation and cubic spline interpolation are used to interpolate an original signal, and the result is shown in Fig. 1.

It can be seen from Fig. 1 that cubic spline interpolation has some errors, and the obtained curve is relatively smooth. The result of piecewise linear interpolation is close to the original function curve as a whole.

In terms of signal similarity before and after interpolation, piecewise linear interpolation achieves better performance. Therefore, this letter chooses piecewise linear interpolation to improve the signal to noise ratio (SNR).

\section{Method}

The advantage of piecewise linear interpolation is that it can overcome the non-convergence of Lagrange interpolation. In fact, piecewise linear interpolation performs linear interpolation by adjacent interpolation base points, and the two points are connected with a straight line, forming a broken line as piecewise linear interpolation function. To calculate the interpolation of point $x$, only two points around $x$ are used, and the amount of calculation is independent of the number of nodes. The principle of piecewise linear interpolation is shown in Fig. 2.

Assuming that the two nodes are $\left(x_{1}, f\left(x_{1}\right)\right)$ and $\left(x_{2}, f\left(x_{2}\right)\right)$, the linear equation on the interval can be written as

$$
y=f(x)=\frac{x-x_{2}}{x_{1}-x_{2}} f\left(x_{1}\right)+\frac{x-x_{1}}{x_{2}-x_{1}} f\left(x_{2}\right) .
$$

\footnotetext{
${ }^{1}$ College of Computer and Information, Hohai University, Nanjing, Jiangsu 210098, China ${ }^{2}$ Faculty of Electronic Information Engineering, Huaiyin Institute of Technology, Huaian, Jiangsu 223003, China, jiayinjie@hhu.edu.cn
} 


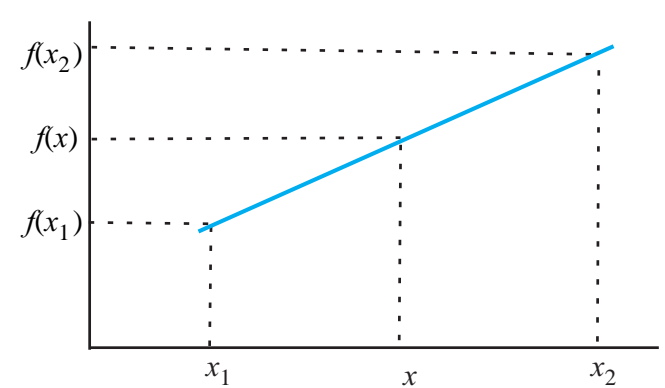

Fig. 2. Piecewise linear interpolation

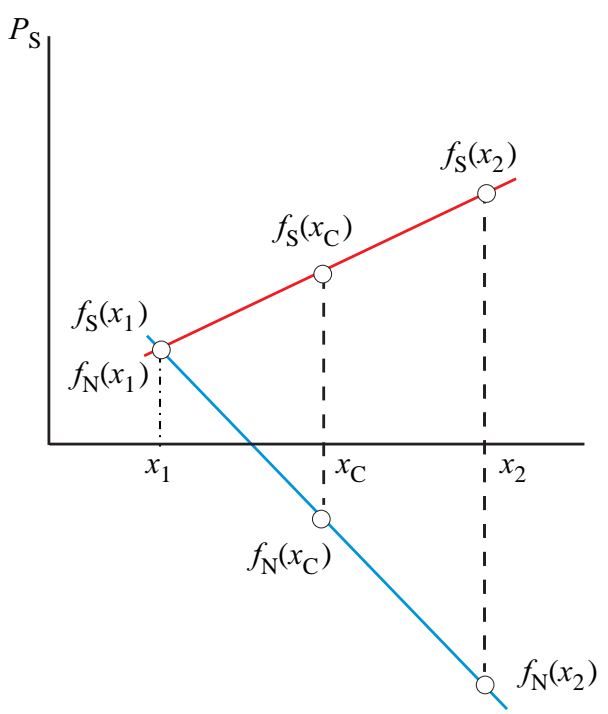

Fig. 3. Piecewise linear interpolation of signal and noise

It can be seen from the above description that the principle of piecewise linear interpolation is very simple. Also, piecewise linear interpolation is featured with less computation, smaller interpolation error, and continuity of interpolation function. However, because the slope at the known point is constant, the result of this interpolation method is not smooth and there are corners. Besides, the interpolation accuracy can be improved by increasing the interpolation points. Geometrically, piecewise linear interpolation connects the interpolation points to form line segments approximate to the original curve, which is also the basic principle of computer graphics. Interpolation can improve the SNR of mixed signal by improving the signal resolution.

According to the fact that the common signal has short-term stationary characteristics, the noise generally does not have stationary characteristics because it is random signal. For the convenience, only two moments, $i e$, $x_{1}$ and $x_{2}$ are considered:

The values of signal $S$, before interpolation are $f_{\mathrm{S}}\left(x_{1}\right)$ and $f_{\mathrm{S}}\left(x_{2}\right)$ in the case of large probability, due to the a short-term stationary characteristic. The values of noise $N$, before interpolation are $f_{\mathrm{N}}\left(x_{1}\right)$ and $f_{\mathrm{N}}\left(x_{2}\right)$ in the case of large probability, due to the non-short-term stationary characteristic. The power of signal $S$ is: $P_{\mathrm{S}}=f_{\mathrm{S}}^{2}\left(x_{1}\right)+$ $f_{\mathrm{S}}^{2}\left(x_{2}\right)$. If for simplicity we assume $f_{\mathrm{S}}\left(x_{1}\right)=f_{\mathrm{N}}\left(x_{1}\right)$ and
$f_{\mathrm{S}}\left(x_{2}\right)=-f_{\mathrm{N}}\left(x_{2}\right)$ we have $P_{\mathrm{S}}=P_{\mathrm{N}}$. This is shown in Fig. 3.

At this time, the initial power of signal $S$ and noise $N$ are equal. Additive white Gaussian noise (AWGN) is one of the basic noise and interference models in communication systems $[5,6]$. Additive noise is a kind of noise superimposed on the signal and here as $N(t)$ always exists with or without the signal. In other words, when signal $S$ passes through the channel with SNR of $0 \mathrm{~dB}$, the power of superimposed noise $P_{\mathrm{N}}$ is equal to the power of signal $P_{\mathrm{S}}$.

The power of signal after piecewise linear interpolation is

$$
P_{\mathrm{SC}}=f_{\mathrm{S}}^{2}\left(x_{1}\right)+f_{\mathrm{S}}^{2}\left(x_{\mathrm{c}}\right)+f_{\mathrm{S}}^{2}\left(x_{2}\right)>P_{\mathrm{S}}
$$

The power of noise after piecewise linear interpolation is

$$
P_{\mathrm{NC}}=f_{\mathrm{N}}^{2}\left(x_{1}\right)+f_{\mathrm{N}}^{2}\left(x_{\mathrm{c}}\right)+f_{\mathrm{N}}^{2}\left(x_{2}\right)>P_{\mathrm{N}}
$$

Since, $\left|f_{\mathrm{N}}\left(x_{c}\right)\right|<\left|f_{\mathrm{S}}\left(x_{c}\right)\right|$, and $\left|f_{\mathrm{N}}\left(x_{1}\right)\right|=\left|f_{\mathrm{S}}\left(x_{1}\right)\right|$, and $\left|f_{\mathrm{N}}\left(x_{2}\right)\right|=\left|f_{\mathrm{S}}\left(x_{2}\right)\right|$ it is true that $P_{\mathrm{NC}}<P_{\mathrm{SC}}$.

It can be concluded that:

- for signal $S$ and noise $N$, the power after piecewise linear interpolation will increase

- Under the same interpolation condition, the power increase of signal $S$ is larger than that of noise $N$.

\section{Results}

The above indicates that the piecewise linear interpolation can improve the SNR, the simulation results in this section will further confirm this point. Music signal [7], LFM (Linear Frequency Modulation) signal or chirp signal [8], and Gaussian white noise signal are taken as examples to verify the conclusions of the previous section. These three kinds of signals respectively conform to super- Gaussian distribution, sub-Gaussian distribution, and Gaussian distribution.

\section{Simulation 1:}

Music signal $S$ passes through AWGN channel with SNR of $0 \mathrm{~dB}$. Also, superimposed noise $N$ and signal $S$ have equal power. The waveform of music signal and superimposed channel noise is shown in Fig. 4.

Figure 5 shows the power comparison of music signal and superimposed channel noise at different multiples of interpolations.

It can be seen from Fig. 5 that when interpolation is not applied (interpolation multiple is 1 ), the power of music signal and white noise is equal. When the interpolation multiple gradually increases from 2 to 5 , the power of music signal and white noise also increase. Also, the increase of white noise power is not as significant as that of music signal.

The increasing gap leads to a larger signal-to-noise ratio, which suppresses the noise.

\section{Simulation 2:}

LFM signal $S$ passes through AWGN channel with SNR of $0 \mathrm{~dB}$. Also, superimposed noise $N$ and signal 

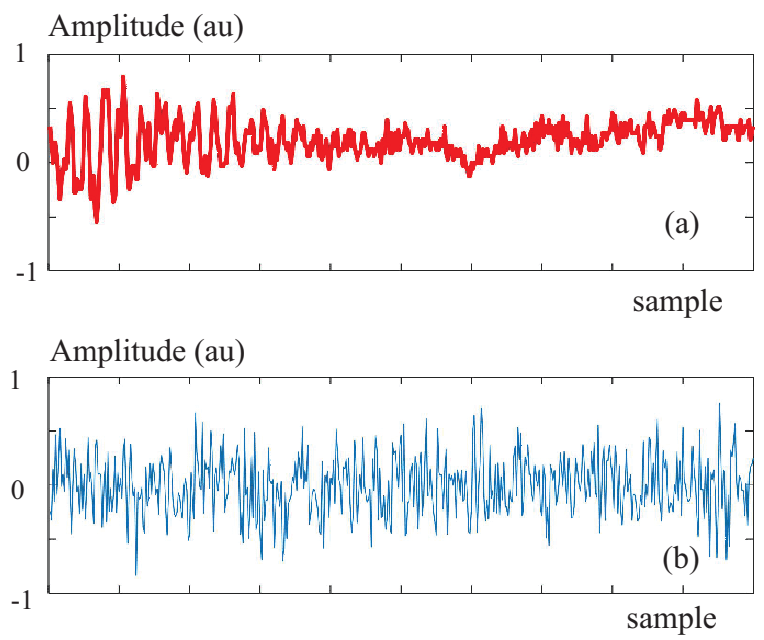

Fig. 4. (a) - music signal, and (b) - white noise

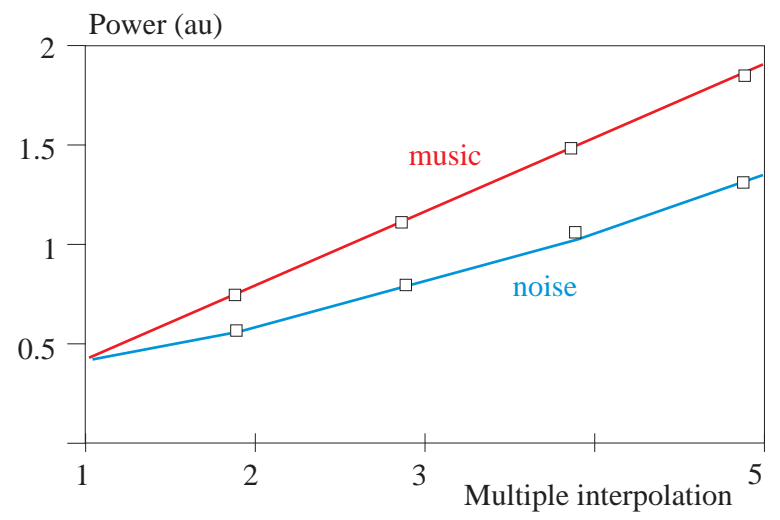

Fig. 5. Power comparison of music signal and white noise at different multiples of interpolation

$S$ have equal power. The waveform of LFM signal and superimposed channel noise is shown in Fig. 6.

Figure 7 shows the power comparison of LFM signal and superimposed channel noise at different multiples of interpolation.

It can be seen from Fig. 7 that when interpolation is not applied (interpolation multiple is 1 ), the power of LFM signal and white noise is equal. When the interpolation multiple gradually increases from 2 to 5 , the power of LFM signal and white noise also increase, but the increase of white noise power is not as significant as that of LFM signal. A larger gap corresponds to an increased signal-to-noise ratio. In this case, the noise reduction is achieved.

\section{Simulation 3:}

Noise signal $S$ passes through AWGN channel with SNR of $0 \mathrm{~dB}$. Also, superimposed noise $N$ and signal $S$ have equal power. The waveform of noise signal and superimposed channel noise is shown in Fig. 8.

Figure 9 shows the power comparison of noise signal and superimposed channel noise at different multiples of interpolation.

It can be seen from Fig. 9 that when the interpolation is not performed (interpolation multiple is 1 ), the power of white noise superimposed on the noise source signal
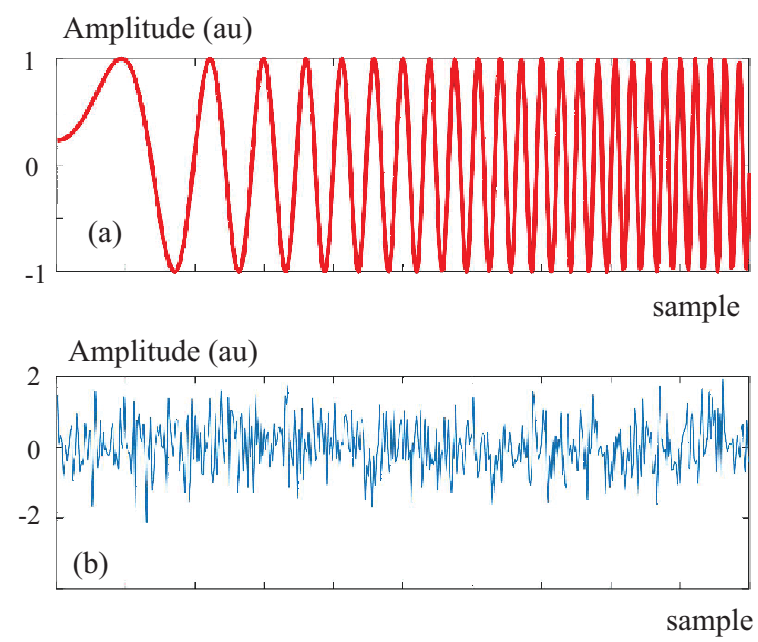

Fig. 6. (a) - LFM signal, and (b) - white noise

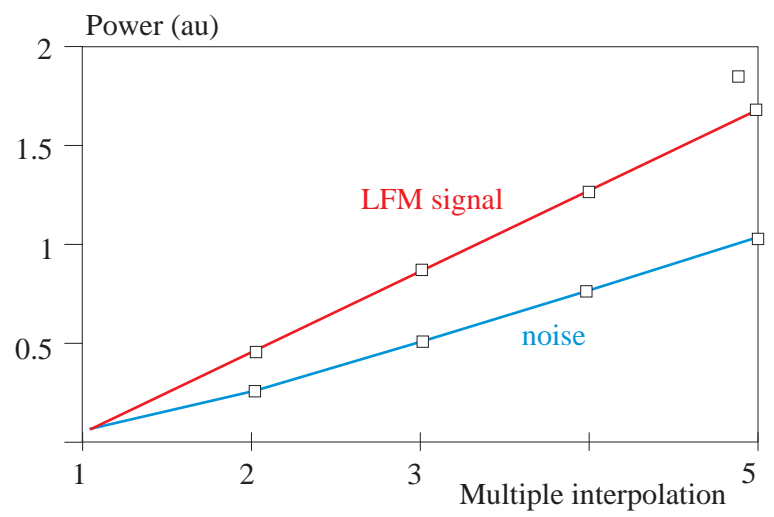

Fig. 7. Power comparison of LFM signal and white noise at different multiples of interpolation

and channel is approximately equal. When the interpolation multiple increases from 2 to 5 , the power of the noise source signal and the white noise increase in an identical trend.

If the signal-to-noise ratio is expressed in $\mathrm{dB}$, the signal-tonoise ratio can be represented as

$$
S N R=10 \log \left(P_{\mathrm{SC}} / P_{\mathrm{NC}}\right) .
$$

Taking simulation 2 as an example, the signal-to-noise of different interpolation is shown in Fig. 10.

It can be seen that when the interpolation is not performed (interpolation multiple is 1), the power of the source signal and the white noise are approximately equal, and the SNR is $0 \mathrm{~dB}$. When the interpolation multiple increases from 2 to 5 , the signal-to-noise ratio also increases, but the increase has a certain limit. With the increase of the interpolation multiple, the signal-to-noise ratio exhibits a slower increase, and it finally reaches a stable value.

\section{Conclusions}

This paper proves for the first time that the piecewise linear interpolation can improve the signal-to-noise ratio. 
Amplitude (au)

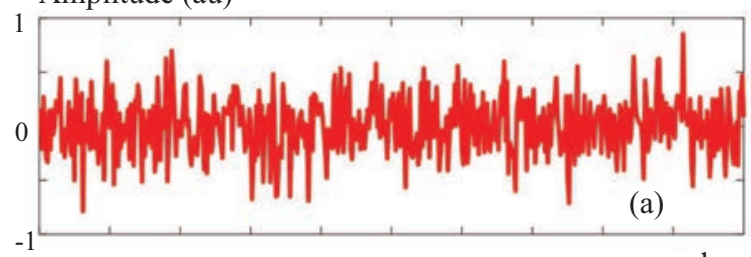

sample

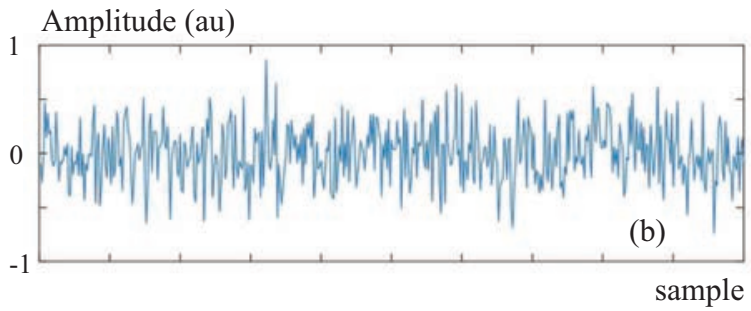

Fig. 8. Noise signal and white noise signal

Also, it is indicated that the piecewise linear interpolation facilitates signal enhancement. Therefore, it can be applied to signal detection, blind source separation, and other fields to preprocess the correlated signals. For example, reasonable interpolation is conducive to improve the performance of blind source separation of mixed signals.

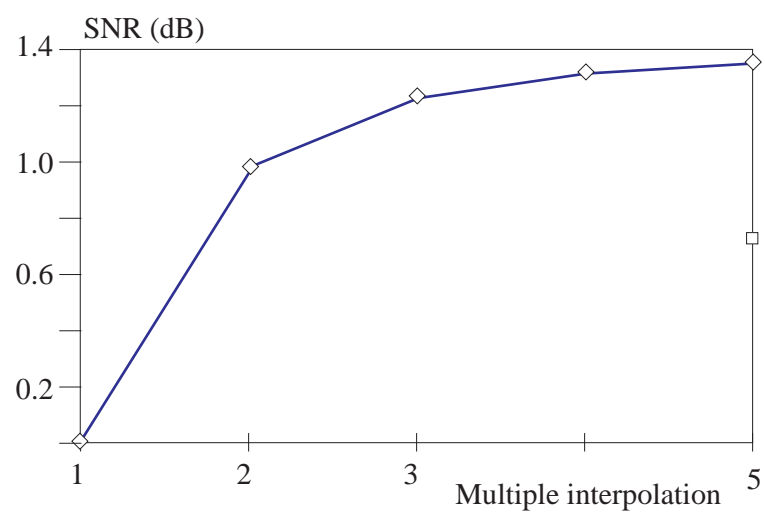

Fig. 10. Power comparison of LFM signal and white noise at different multiples of interpolation

\section{Acknowledgements}

This work was partially supported by the project of industrial-academic-research cooperation of jiangsu province (No.BY2020241)

\section{REFERENCES}

[1] V. V. Marchencov, T. V. Saveleva, and V. A. Solovei, "An interpolation method in measurements of time intervals via their direct coding", Instruments and experimental techniques, 58(3), 364-366 (2015).

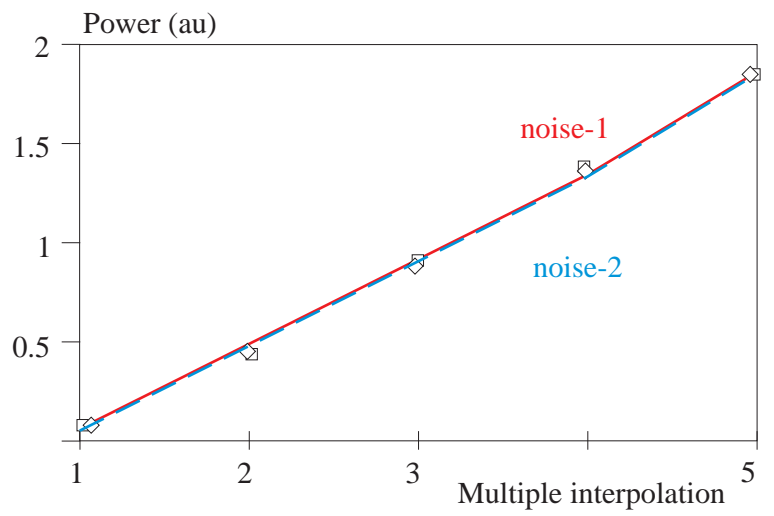

Fig. 9. Power comparison of music signal and white noise at different multiples of interpolation

[2] A. Kumar and J. Haupt, "Convexifying sparse interpolation with infinitely wide neural networks: an atomic norm approach", IEEE Communications Letters, 24(12), 2114-2118 (2020).

[3] P. Manfredi, I. S. Stievano, and F. G. Canavero, "Efficient prediction of stochastic eye patterns via lagrange interpolation", Electronics letters, 49(25), 1615-1617 (2013).

[4] D. Lamb, L. F. O. Chamon, and V. H. Nascimento, : "Efficient filtering structure for spline interpolation and decimation", Electronics letters, 52(1), 39-41 (2016).

[5] P. Xu, Y. Jia, Z. Wang, and M. Jiang, "Underdetermined blind source separation for sparse signals based on the law of large numbers and minimum intersection angle rule", Circuits systems and signal processing, 39(5), 2442-2458 (2020).

[6] Y. Jia and P. Xu, "Convolutive Blind Source Separation for communication signals based on the sliding Z-transform", IEEE Access, 8, 41213-41219 (2020).

[7] P. Xu, Y. Jia, and M. Jiang, "Blind audio source separation based on a new system model and the savitzky-golay filter", Journal of Electrical Engineering, 72(3), 1-5 (2021).

[8] P. Xu and Y. Jia, "Blind source separation for chirp signals based on the local quadratic regression smoothing", Comptes rendus de l'Acadmie bulgare des Sciences, 73(11), 1579-1585 (2020).

Received 24 September 2021

Pengfei $\mathrm{Xu}$ received the MS degree in communication and information systems from the Xidian University,China,in 2010, now working towards the $\mathrm{PhD}$ degree in computer science and technology at Hohai University. His research interests include signal processing, machine learning and compressive sensing.He authored and coauthored eleven articles.

Yinjie Jia received the MS degree in communication and information systems from the Xi'an University of Science and Technology,China, in 2011. She is now working towards the $\mathrm{PhD}$ degree in computer science and technology at Hohai University. Her research interests include signal processing, machine learning and compressive sensing. She authored and coauthored six articles. 